\title{
Numerical simulations of impulsively generated vertical oscillations in a solar coronal arcade loop
}

\author{
M. Selwa ${ }^{1}$, S. K. Solanki ${ }^{2}$, K. Murawski ${ }^{1}$, T. J. Wang ${ }^{2}$, and U. Shumlak ${ }^{3}$ \\ ${ }^{1}$ Group of Astrophysics and Gravity Theory, Institute of Physics, UMCS, ul. Radziszewskiego 10, 20-031 Lublin, Poland \\ e-mail: mselwa@kft.umcs. lublin.pl \\ 2 Max-Planck-Institut für Sonnensystemforschung, Max-Planck-Str. 2, 37191 Katlenburg-Lindau, Germany \\ 3 Aeronautics and Astronautics Department, University of Washington, Box 352250, Seattle, WA 98195-2250, USA
}

Received 30 September 2005 / Accepted 22 February 2006

\begin{abstract}
Aims. The main aims of the paper are to carry out numerical simulations of the vertical oscillations in a coronal loop in order to determine their dependence on various parameters and to compare them with recent TRACE observations.

Methods. We consider impulsively generated oscillations in a solar coronal arcade loop. The two-dimensional numerical model we implement in the ideal MHD regime includes the effects of nonlinearity and line curvature. We perform parametric studies by varying both the position and the width/strength of the pulse.

Results. A pulse launched below a loop is in general found to excite multiple wave modes, in particular a vertical oscillation with many properties of a kink mode, fast mode oscillations and a slow mode pulse (or two slow mode pulses, depending on the location of the original pulse). From our parametric studies we deduce that wave periods and attenuation times of the excited waves depend on the position below the loop summit, as well as on the width of the pulse. Wider pulses launched closer to a foot-point and to the loop's apex trigger wave packets of longer period waves which are more strongly attenuated. A perturbed loop does not return to its initial state but is instead stretched, with its apex shifted upwards. As a result the perturbations propagate along the stretched loop and consequently stronger and wider pulses which stretch a loop more lead to longer period oscillations. A pulse located near one of the foot-points is found to excite a distortion mode leading to asymmetric oscillations which are distinct from the vertical or horizontal kink modes that have been identified in TRACE data.
\end{abstract}

Key words. magnetohydrodynamics (MHD) - Sun: corona - Sun: oscillations

\section{Introduction}

Magnetic loops are the main ingredients of the solar corona that sustain oscillations (e.g., Nakariakov et al. 1999; Aschwanden et al. 1999; Wang et al. 2002; de Moortel et al. 2002). In one of the more recent observations Wang \& Solanki (2004) reported on vertical oscillations of a 300-400 Mm long solar coronal loop which expanded and shrank with an oscillation period of $\approx 230 \mathrm{~s}$. They also found that the oscillating loop was associated with intensity variations which suggested that these oscillations were compressible. Here we concentrate on modeling this mode of oscillation.

Theoretical studies of loop oscillations are numerous. Analytical studies were done by, e.g., Roberts et al. (1984), Nakariakov et al. (1999), Nakariakov (2003), Van Doorsselaere et al. (2004), while numerical simulations were performed by Murawski \& Roberts (1993), Smith et al. (1997), Murawski et al. (2005a,b), del Zanna et al. (2005), Brady \& Arber (2005).

In particular, Nakariakov et al. (1999) estimated for the first time the decay time of the transverse oscillations, and its ratio to the period. Transverse oscillations in solar coronal loops induced by propagating Alfvénic pulses were discussed by del Zanna et al. (2005). Fast standing waves were numerically simulated by Murawski et al. (2005a) who found high-order standing fast kink mode oscillations. The main drawback of their model was that it corresponds to an unrealistically high $\beta$ in the circular coronal loop. Another shortcoming of this paper is a simple model of the photosphere, described by dense plasma layer located at the loop foot-points. Although all boundary conditions were set open the dense layer reflected waves back into a rarefied region. This drawback was removed by Murawski et al. (2005b), who showed that impulsively triggered fast magnetosonic waves in a cool loop (with a temperature of 1-2 MK) have periods which are compatible with the observational data provided by TRACE which may be interpreted as a fast kink mode in an arcade. In the adopted model line-tying boundary conditions were set at the loop foot-points to reflect fast magnetosonic waves that were only present in the considered system. Selwa et al. (2005b) (hereafter Paper I) extended this model to a strongly magnetized coronal loop with a low plasma $\beta$, allowing the slow wave to be present in the system. They found a number of features in common with the observations. However, they discussed only a single example of a pulse launched centrally at the photospheric level, while parametric studies are required to understand the complex scenario of loop oscillations. They applied line-tying boundary conditions at the left and right sides of a simulation region. Such a choice of boundary conditions are not completely physically justified.

The main goal of this paper is to perform parametric studies and improve on the models of Murawski et al. (2005a,b) and Selwa et al. (2005b). In addition, since we employ a different code we can also test to what extent the previous results are influenced by numerical limitations. 
The paper is organized as follows: the numerical model is described in Sect. 2. The numerical results are presented and discussed in Sect. 3 and the paper is concluded by a short summary of the main results in Sect. 4.

\section{Numerical model}

We describe coronal plasma by the ideal magnetohydrodynamic (MHD) equations:

$$
\begin{aligned}
& \frac{\partial \varrho}{\partial t}+\nabla \cdot(\varrho \boldsymbol{V})=0, \\
& \frac{\partial(\varrho \boldsymbol{V})}{\partial t}+\nabla \cdot[(\varrho \boldsymbol{V}) \boldsymbol{V}]=-\nabla p+\frac{1}{\mu}(\nabla \times \boldsymbol{B}) \times \boldsymbol{B}, \\
& \frac{\partial E}{\partial t}+\nabla \cdot\left[\left(E+p_{\mathrm{T}}\right) \boldsymbol{V}-\frac{\boldsymbol{B}(\boldsymbol{B} \cdot \boldsymbol{V})}{\mu}\right]=0, \\
& \frac{\partial \boldsymbol{B}}{\partial t}=\nabla \times(\boldsymbol{V} \times \boldsymbol{B}), \\
& \nabla \cdot \boldsymbol{B}=0 .
\end{aligned}
$$

Here $\mu$ is the magnetic permeability, $\varrho$ is mass density, $\boldsymbol{V}$ is flow velocity, $p$ is gas pressure, $\boldsymbol{B}$ is magnetic field, the symbol $p_{\mathrm{T}}$ denotes the total pressure that represents the sum of the gas and magnetic pressures:

$p_{\mathrm{T}}=p+\frac{B^{2}}{2 \mu}$

and plasma energy density is expressed as

$E=\frac{\varrho V^{2}}{2}+\frac{p}{\gamma-1}+\frac{B^{2}}{2 \mu}$,

where $\gamma$ is the adiabatic index.

\subsection{Equilibrium configuration}

We adopt and modify the coronal arcade model that was recently described in Paper I. Earlier wave studies, in which this magnetic arcade configuration was used, were performed by e.g. Oliver et al. (1996), Cadez et al. (1996), Oliver et al. (1998), Terradas et al. (1999). In this model the coronal arcade is settled in a two-dimensional, gravity-free and motionless environment. The equilibrium magnetic field $\boldsymbol{B}_{\mathrm{e}}=\left[B_{\mathrm{e} x}, 0, B_{\mathrm{e} z}\right]$ has two nonzero components which are specified with the help of the vector magnetic potential $\boldsymbol{A}=A \hat{\boldsymbol{y}}$ as

$\boldsymbol{B}_{\mathrm{e}}=\nabla A \times \hat{\boldsymbol{y}}$,

where $\hat{\boldsymbol{y}}$ is a unit vector along the $y$-direction and $A$ satisfies Laplace's equation, $\nabla^{2} A=0$, whose solution is

$A(x, z)=B_{0} \Lambda_{\mathrm{B}} \cos \left(x / \Lambda_{\mathrm{B}}\right) \mathrm{e}^{-z / \Lambda_{\mathrm{B}}}$.

From Eq. (8) we obtain then

$$
\begin{aligned}
& B_{\mathrm{e} x}=B_{0} \cos \left(x / \Lambda_{\mathrm{B}}\right) \mathrm{e}^{-z / \Lambda_{\mathrm{B}}}, \\
& B_{\mathrm{e} z}=-B_{0} \sin \left(x / \Lambda_{\mathrm{B}}\right) \mathrm{e}^{-z / \Lambda_{\mathrm{B}}},
\end{aligned}
$$

where $B_{0}$ is the magnetic field at the level $z=0$ and $\Lambda_{\mathrm{B}}$ is the magnetic scale height such that

$\Lambda_{\mathrm{B}}=\frac{2 L}{\pi}$.

Here $L$ is the horizontal half-width of the arcade, chosen as $L=100 \mathrm{Mm}$.

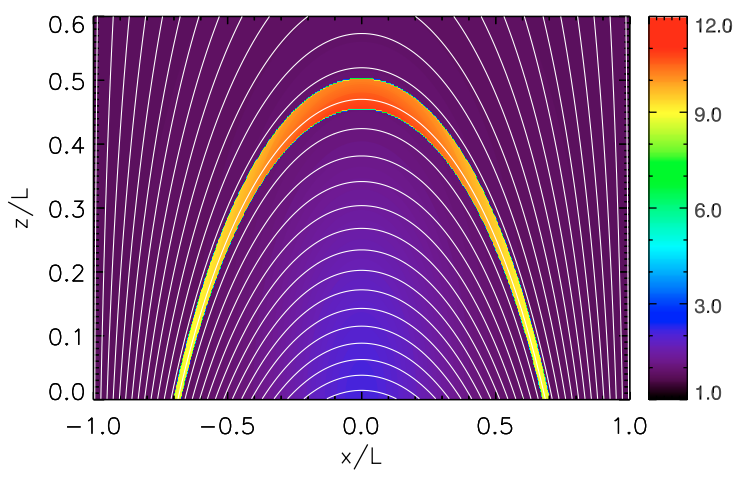

Fig. 1. Initial configuration (time $t=0$ ) with a pulse centered at $x_{0}=$ $z_{0}=0$ with $w=0.35 \mathrm{~L}$. The density (colour bar) is given in arbitrary units $\left(10^{-12} \mathrm{~kg} \mathrm{~m}^{-3}\right)$. The loop is seen as a region of higher density. Magnetic field lines are shown as solid white lines. Note that the loop's apex is denser than the foot-points due to the hot dense pulse.

For the background Alfvén speed we have chosen $V_{\mathrm{Ae}}=$ $\left|B_{\mathrm{e}}\right| / \sqrt{\mu \varrho_{\mathrm{e}}}$, where $\varrho_{\mathrm{e}}$ is the background mass density. In this case $V_{\text {Ae }}$ decays exponentially with height $z$ and the background temperature $T_{\mathrm{e}} \sim p_{\mathrm{e}} / \varrho_{\mathrm{e}}$ is constant. Here $p_{\mathrm{e}}=$ const. is the background pressure.

Next we embed a loop, i.e. a group of field lines supporting gas with higher density, in the arcade in such a way that its edges follow two specific magnetic field lines. We prescribe that the inner and outer field lines cross the base of the arcade at $|x|=$ $L_{\mathrm{f}}-2 a_{\mathrm{f}}$ and $|x|=L_{\mathrm{f}}$, respectively. Inside the loop we use the following density profile:

$\varrho(x, z)=d \varrho_{\mathrm{e}}\left[H\left(\frac{A-A_{2}}{B_{0} \Lambda_{\mathrm{B}}}\right)-H\left(\frac{A-A_{1}}{B_{0} \Lambda_{\mathrm{B}}}\right)\right]$,

where $A_{1}=A\left(L_{\mathrm{f}}, 0\right)<A_{2}=A\left(L_{\mathrm{f}}-2 a_{\mathrm{f}}, 0\right)$ and $H$ is Heaviside's function. The mass density in this loop varies abruptly at its sides, leading to a density enhancement of the loop compared to the ambient medium. We choose the mass density contrast $d=\varrho_{\mathrm{i}} / \varrho_{\mathrm{e}}=10$, with $\varrho_{\mathrm{i}}$ and $\varrho_{\mathrm{e}}$ corresponding to mass density within the loop and in the ambient medium, respectively. As a reference we set $L_{\mathrm{f}}=0.7 L$ and $a_{\mathrm{f}}=0.0125 L$ : they uniquely specify the loop's length $l$, height $h$ and its width $2 a$ at the summit. This loop does not have a perfect circular shape, but its average radius and length can be estimated as $70 \mathrm{Mm}$ and $190 \mathrm{Mm}$, respectively (Fig. 1; note the difference in horizontal and vertical scale).

Due to the enhanced density, the Alfvén speed within the loop is smaller than in the ambient medium. As a result of this depression in the Alfvén speed the loop becomes a cavity for fast magnetosonic waves. We denote the Alfvén speed within the loop by $V_{\mathrm{Ai}}=V_{\mathrm{Ae}} / \sqrt{d}$.

The magnetic field at the photospheric level, $B_{0}$, is connected to the reference mass density $\varrho_{\mathrm{e}}$ through the reference Alfvén speed $V_{\mathrm{A} 0}=B_{0} / \sqrt{\mu \varrho_{\mathrm{e}}(0)}$, where we assume $\varrho_{\mathrm{e}}=10^{-12} \mathrm{~kg} \mathrm{~m}^{-3}$ and $V_{\mathrm{A} 0}=1 \mathrm{Mm} \mathrm{s}^{-1}$ for the mass density and the Alfvén speed, respectively, at the level $z=0$.

For a potential magnetic arcade the equilibrium pressure $p_{\mathrm{e}}$ has to be constant. This pressure can be evaluated from the definition of the plasma $\beta=2 \mu p_{\mathrm{e}} / B_{\mathrm{e}}^{2}$, adopted at the level $z=0$. In this model $\beta$ grows from 0.012 at the loop foot-points to 0.054 at the loop's apex. This growth is due to the absence of gravity in the potential-field structure and is not realistic. However, the value of $\beta$ remains below unity. 


\subsection{Perturbations}

Perturbations in Eqs. (1)-(3) can be excited in such a loop in numerous ways. As we are interested in impulsively excited waves, we launch a hot pulse in the pressure and mass density, i.e.

$\delta \varrho(x, z, t=0)=A_{\varrho} \varrho_{\mathrm{e}} \mathrm{e}^{-\left(\left(x-x_{0}\right)^{2}+\left(z-z_{0}\right)^{2}\right) / w^{2}}$,

$\delta p(x, z, t=0)=A_{\mathrm{p}} p_{\mathrm{e}} \mathrm{e}^{-\left(\left(x-x_{0}\right)^{2}+\left(z-z_{0}\right)^{2}\right) / w^{2}}$,

where $w$ is the initial pulse width and $\left(x_{0}, z_{0}\right)$ denotes its initial position. We choose initial relative amplitudes of the pulse $A_{\varrho}=$ $A_{\mathrm{p}} / 10$ which corresponds to a pulse that is 6.4 times hotter than the average, unperturbed corona. It is noteworthy that the vertical oscillations seen by TRACE (Wang \& Solanki 2004) are identified in gas at around $1 \mathrm{MK}$. We have no information of the temperature of the pulse causing this oscillation, although the gas in the oscillating loop does not appear to get significantly heated by the pulse. The above initial disturbance is taken to be a hot pulse, mimicking a flare. Kink oscillations are often reported to be excited by passing blast waves or nearby eruptive events, so that a kinematic pulse (in velocity and density) could be more suitable. We have verified that the outcome of the simulations does not significantly depend on which of the two types of impulsive excitations is chosen. In the absence of further information we adopt the above values of the amplitudes, which had been successfully employed in Paper I, as reference values.

\section{Numerical results}

The numerical code EMILY we adopt was developed by Jones et al. (1997). This code employs an explicit-implicit algorithm for solving the time-dependent, non-ideal magnetohydrodynamic equations. The algorithm is a finite-volume scheme that uses an approximate Riemann solver for the hyperbolic fluxes and central differencing applied on nested control volumes for the parabolic fluxes that arise from the non-ideal terms (i.e., resistivity and viscosity). This scheme is second-order accurate in space and time. In our studies we used the explicit option of the code for ideal magnetohydrodynamic equations.

Equations (1)-(5) are solved numerically in an Eulerian box with the $x$ - and $z$-dimensions $(-L, L) \times(0,2 L)$. This box is covered by a uniform grid of $300 \times 400$ or $600 \times 800$ numerical cells. Grid convergence studies, which are based on grid refinement, are performed to show that the numerical results are not affected by insufficient spatial resolution. We apply open boundary conditions, with a zero-gradient extrapolation of all plasma variables, at the left, right and top sides of the simulation region, thus allowing a wave signal to leave freely the simulation region. As the adopted numerical method is based on characteristics the open boundaries indeed let all types of MHD waves pass through. Numerically induced reflections from such boundaries have been found to be small. We set line-tying boundary conditions at the bottom of the simulation region. These boundaries model the interaction between the denser photosphere and the overlying plasma layers.

\subsection{Pulses of various amplitudes}

We begin our parametric studies with variations of the amplitude of the initial pulse, $A_{\mathrm{p}}$, while holding the initial position $x_{0}=$ $z_{0}=0$ and the pulse width $w=35 \mathrm{Mm}$ fixed.

Figure 2 displays time-signatures of the mass density along the line $x=0$ at heights close to the apex of the loop for $A_{\mathrm{p}}=15 p_{\mathrm{e}}$ (top panel) and $A_{\mathrm{p}}=30 p_{\mathrm{e}}$ (bottom panel).
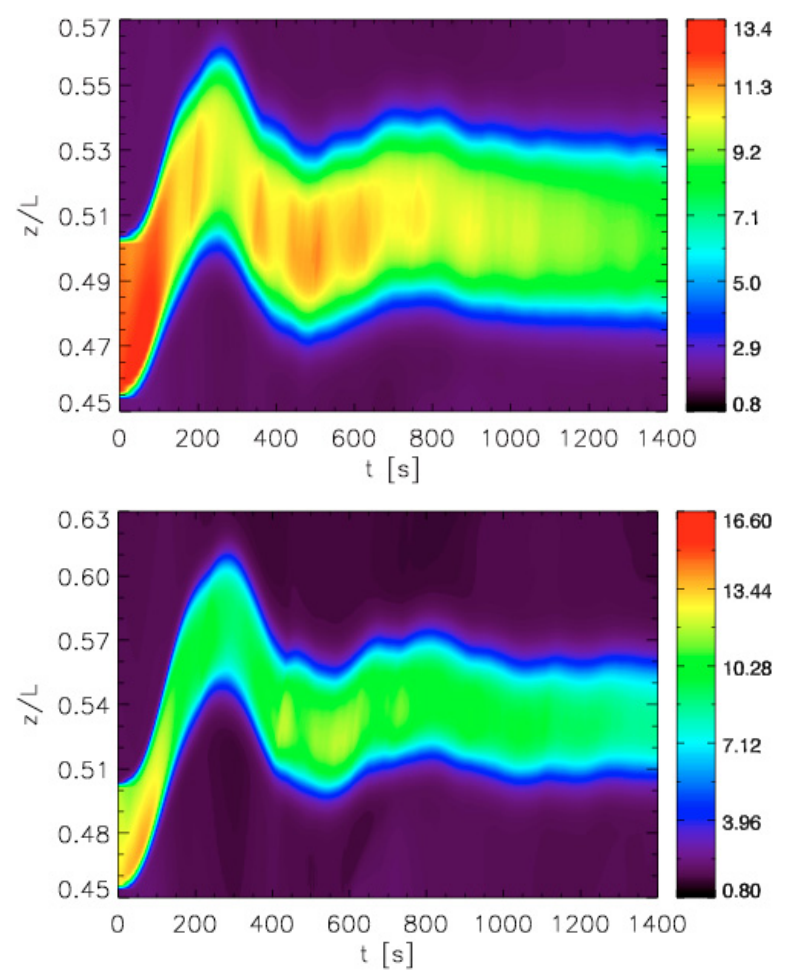

Fig. 2. Time-signatures of the mass density (colour scale; arbitrary units: $10^{-12} \mathrm{~kg} \mathrm{~m}^{-3}$ ) along a vertical cut through the loop's apex for pulse amplitude $A_{\mathrm{p}}=15 p_{\mathrm{e}}$ (top panel) and $A_{\mathrm{p}}=30 p_{\mathrm{e}}$ (bottom panel). Spatial coordinates and time are measured in units of $L$ and in seconds, respectively.

The moment $t=0$ corresponds to the time at which the pulse is released. As a result of their higher energies stronger pulses lead to more significant initial shifts of the loop summit. The loop's apex is initially displaced upward by $\sim 0.06 L$ (top panel) and $\sim 0.12 L$ (bottom panel) which for the chosen value of $L=10^{2} \mathrm{Mm}$ correspond to $\sim 6 \mathrm{Mm}$ and $\sim 12 \mathrm{Mm}$, respectively. The observed displacement of 7.9 Mm (Wang \& Solanki 2004) lies between these values. Thus, in contrast to the event simulated in Paper I, we can obtain similar displacements as observed.

Except for the amplitude of the displacement the strength of the pulse does not have a qualitalively different effect in the two cases, although there are some quantitative differences, as discussed later in this section. Both loops display a similar reduction in density at the apex. It is noteworthy that the apex position along the $z$-axis and density at the apex are anticorrelated (with correlation coefficient -0.71) in general agreement with the observations. Note also the rapid oscillations with smaller amplitude, which are also partly visible in the density (mainly in the upper frame). These were identified as sausage mode oscillations in Paper I. This identification is confirmed by our simulation based on a different numerical code.

It is noteworthy that the loop does not return to its initial position, once the oscillations have subsided, displaying an offset which is larger for larger pulses (top panel of Fig. 3), being approximately $0.02 \mathrm{~L}$ in the top panel and approximately $0.05 \mathrm{~L}$ in the bottom panel (Fig. 2). This offset is probably a consequence of the loop in the simulations reaching a final equilibrium that is different from the initial one due to the non-potential shape component added by the pulse (the apex goes up and foot-points draw together as the density follows the Gaussian shape of the pulse). However, since the open upper boundary is not capable 


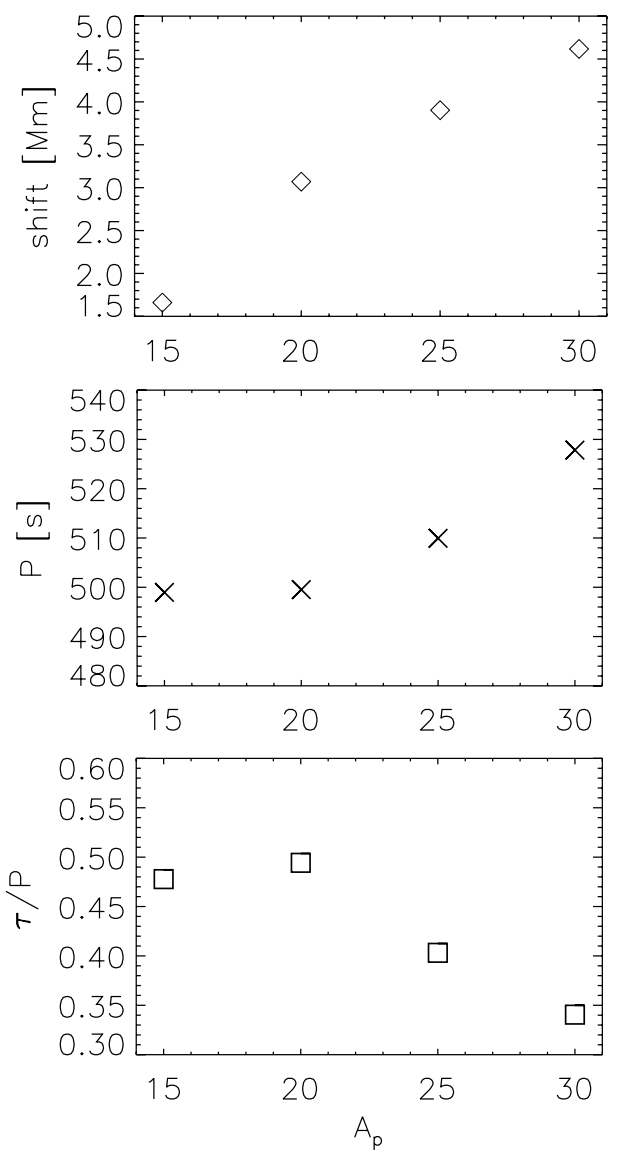

Fig. 3. Final shift of the loop's apex (top panel), period $P$ (middle panel), and the ratio of attenuation time $\tau$ to period, $\tau / P$ (bottom panel) vs. pulse amplitude $A_{\mathrm{p}}$.

of conserving magnetic tension, this does not allow the initial state to be exactly restored. We therefore cannot rule out that the offset, or a part of it, is due to this effect.

The oscillations seen in Fig. 2 represent the response of the loop summit to the initial perturbation. They correspond to a packet of waves among which kink waves exhibit the main contribution (Murawski et al. 2005a,b, Paper I). As the signal in Fig. 2 decays with attenuation time $\tau$, the wave period also evolves with time. However, it is instructive to measure the period when the signal is strongest, $P$. Values of $P$ and of the ratio $\tau / P$ are presented in the middle and bottom panels of Fig. 3 . These periods and attenuation times are obtained by fitting the time-signatures of the simulated kink mode (i.e. vertical location of the loop's apex vs. time) with attenuated sine functions (Paper I). For pulse amplitude $A_{\mathrm{p}}=15 p_{\mathrm{e}}$ the period of the excited vertical oscillation $P \simeq 500 \mathrm{~s}$, while a pulse with double the amplitude leads to $P \simeq 530 \mathrm{~s}$. As a consequence of stronger pulses, larger amplitude and slightly larger period waves are excited. Such larger amplitude pulses shift the loop's apex towards higher altitudes (Fig. 2). As a result the loop becomes stretched, its effective length becomes larger and consequently the wave period attains a higher value. As $\tau / P$ declines from 0.49 for $A_{\mathrm{p}}=15 p_{\mathrm{e}}$ to 0.34 for $A_{\mathrm{p}}=30 p_{\mathrm{e}}$ we conclude that larger amplitude or longer period waves are more strongly attenuated. A detailed study of the cause of this behaviour of the attenuation will be given elsewhere.

The top panel of Fig. 2 is very similar to Fig. 1 of Paper I. However, there are a few differences due to the fact that we use another code. The apparent filamentation of the loop seen in

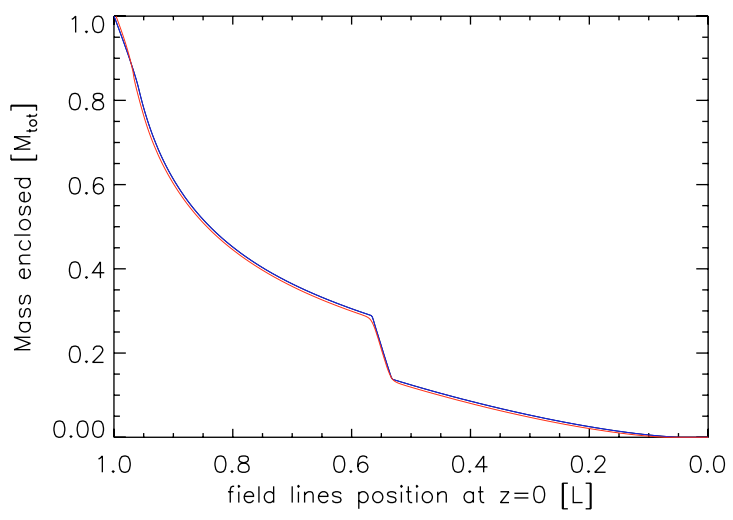

Fig. 4. Mass (measured in total mass units) enclosed in the area bounded by field lines (the position of field lines is indicated by the location on which they cross the $z=0$ line) for a pulse launched at $x_{0}=z_{0}=0$, $w=0.35 L, A_{\mathrm{p}}=15$ for $t=0$ (blue curve) and $t=1400 \mathrm{~s}$ (red curve).

Fig. 1 of Paper I is absent in Fig. 2. This supports the conclusion in Paper I that this filamentation is a numerical artifact. The wave profiles, however, appear to be more smoothed by numerical diffusion in Fig. 2 than in Fig. 1 of Paper I: the loop gets wider and less dense. However, this effect is not excessively large, as we have found from several tests. Thus, Fig. 4 shows the mass enclosed in the area bounded by field lines starting from the outer most one to the one located in the middle of the simulation region. For the initial moment $(t=0$, blue curve) and the end of simulations ( $t=1400 \mathrm{~s}$, red curve) we do not observe much difference in the distribution of mass in this plot. This shows that the numerical diffusion of mass across field lines (recall that we restrict our computations to ideal MHD) is minimal.

Henceforth we choose and hold fixed $A_{\mathrm{p}}=15 p_{\mathrm{e}}$.

Some clarification of the difference between the final and initial states may be useful here. The final magnetic field is nearly current-free, as $\nabla \times \boldsymbol{B}$ remains close to zero. The departure of this value from zero grew with time but remained at an acceptable level. Similarly, $\nabla \cdot \boldsymbol{B}$ remained close to zero everywhere except at the bottom boundary. We specify the normalized divergence error by computing the value $\left|\nabla \cdot \boldsymbol{B}\left(l_{0} / B\right)\right|$, where $l_{0}$ is the spatial scale unit. It allows us to state that the average value throughout the domain is $\sim 10^{-4}$ with two peak values (minimum and maximum) located close to the line $x=0$. The larger value of $\nabla \cdot \boldsymbol{B}$ occurs only at the boundary, as the line-tying boundary conditions are adopted there. These boundary conditions are inconsistent for the magnetic field as we use a zero-gradient extrapolation for it. The underlying assumption is that $\boldsymbol{B}$ is smooth, and accurately computed at the interior points. We have found that grid refinement leads to decreasing the divergence errors without altering the results of the simulation.

As a consequence of the peak value in $\nabla \cdot \boldsymbol{B}$ we observe some numerical errors developing close to the line $x=0$. These errors are particularly apparent in temperature and density profiles for $t>1000 \mathrm{~s}$ and are hardly noticeable prior to that. As these errors arise in the final stage of our simulations, they do not play any significant role in the time signatures of the vertical oscillations, which are attenuated before that time.

As a consequence of the initial hot pulse the plasma was heated up, with most of the excess heat remaining below the loop so that the plasma became compressed in this hot region. This results from the application of the ideal MHD equations in the present model. Thermal conduction, if applied, would be able to redistribute this heat more efficiently along magnetic field lines. 


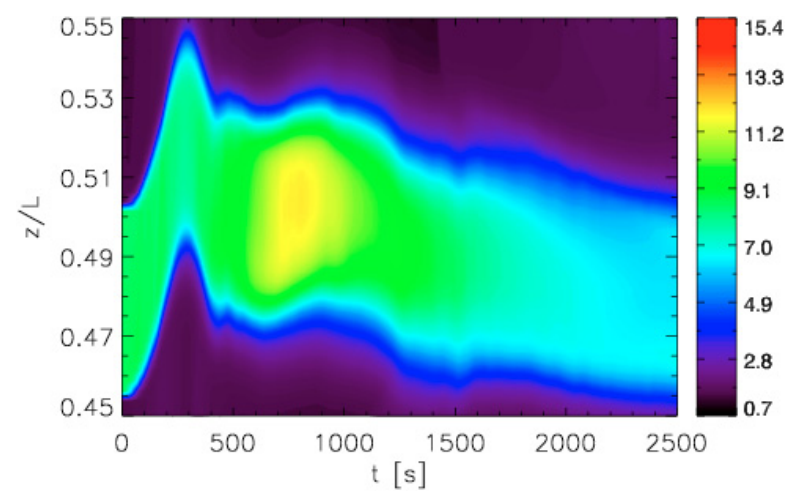

Fig. 5. Time-signature of the mass density (colour scale; arbitrary units: $10^{-12} \mathrm{~kg} \mathrm{~m}^{-3}$ ) triggered by the initial pulse launched at $x_{0}=-0.42 \mathrm{~L}$, $z_{0}=0$. Compare with the top panel of Fig. 2 for which $x_{0}=0$.

\subsection{Dependence on pulse position: pulses along horizontal line $z_{0}=0$}

In this part of the paper we discuss the influence of pulses launched along a horizontal line that joins the two foot-points of the loop, i.e. pulses with initial location $z=0$, but different $x$. The width of the pulse is fixed as $w=0.35 \mathrm{~L}$. Figure 5 shows the time-signature of a vertical cut at $x=0$, encompassing the height of the loop apex in response to a pulse launched in the neighborhood of the left foot-point which is located at $x_{0} / L=-0.7$. Figure 5 shows some similarities but also considerable differences compared to Fig. 2. A kink oscillation, corresponding basically to a single pulse, is visible at $250 \mathrm{~s}<t<600 \mathrm{~s}$. This pulse results from fast magnetosonic waves which reached the detection region while initially propagating outside the loop in the ambient medium. This corresponds to the same excitation mechanism as for the pulses discussed in Sect. 3.1.

For $x_{0} \neq 0$ the kink mode is excited non-symmetrically (not as a simple "breathing-like" motion) and a so-called distortion oscillation is set up. The loop is distorted while nearly maintaining its length. Figure 6 shows three snapshots of a loop for $t=200 \mathrm{~s}, t=300 \mathrm{~s}$ and $t=375 \mathrm{~s}$. It reveals that the main perturbation is a sideways motion of the upper part of the loop, in contrast to the breathing motion found in Sect. 3.1. At later times the loop is more symmetric. Figure 7 shows the shift of the apex along the $x$-axis for a pulse launched at $x_{0}=-0.42 L, z_{0}=0$. The shift denotes the horizontal displacement of the loop's apex. It is noteworthy that the horizontal motion of the loop does not decay as fast as its vertical motion (compare Figs. 5 with 7). Also, the period of horizontal distortion oscillations is slightly shorter ( $452 \mathrm{~s}$ in the case of $x_{0}=-0.42 \mathrm{~L}$, Fig. 7) than the period of vertical ones, so that the ratio of horizontal attenuation time to the period of horizontal oscillations is $\sim 3$ compared to ratios $<1$ for vertical oscillations. Wave period $P$ and ratio $\tau / P$ of the vertical oscillation is plotted vs. normalized pulse position $\left|x_{0} / L_{\mathrm{f}}\right|$ in Fig. 8. It is noteworthy that $P$ grows and $\tau / P$ decreases with $\left|x_{0}\right|$. Hence, an initial pulse launched along $z_{0}=0$ farther out from the symmetrical position $x_{0}=0$ excites longer period vertical waves which are more strongly attenuated.

The density enhancement marked by the orange-yellow spot in Fig. 5 between $t=700 \mathrm{~s}$ and $t=900 \mathrm{~s}$ corresponds to the slow wave that propagates within the loop from the left foot-point towards the loop summit. This time lag is somewhat shorter than the travel time of a slow mode from a foot-point to the apex: the period $l / 2 c_{\mathrm{s}} \approx 950 \mathrm{~s}$, where $l$ is the loop length and $c_{\mathrm{s}}$ denotes the sound speed. Now, the pulse propagates outside the loop at the fast wave speed and excites the slow pulse
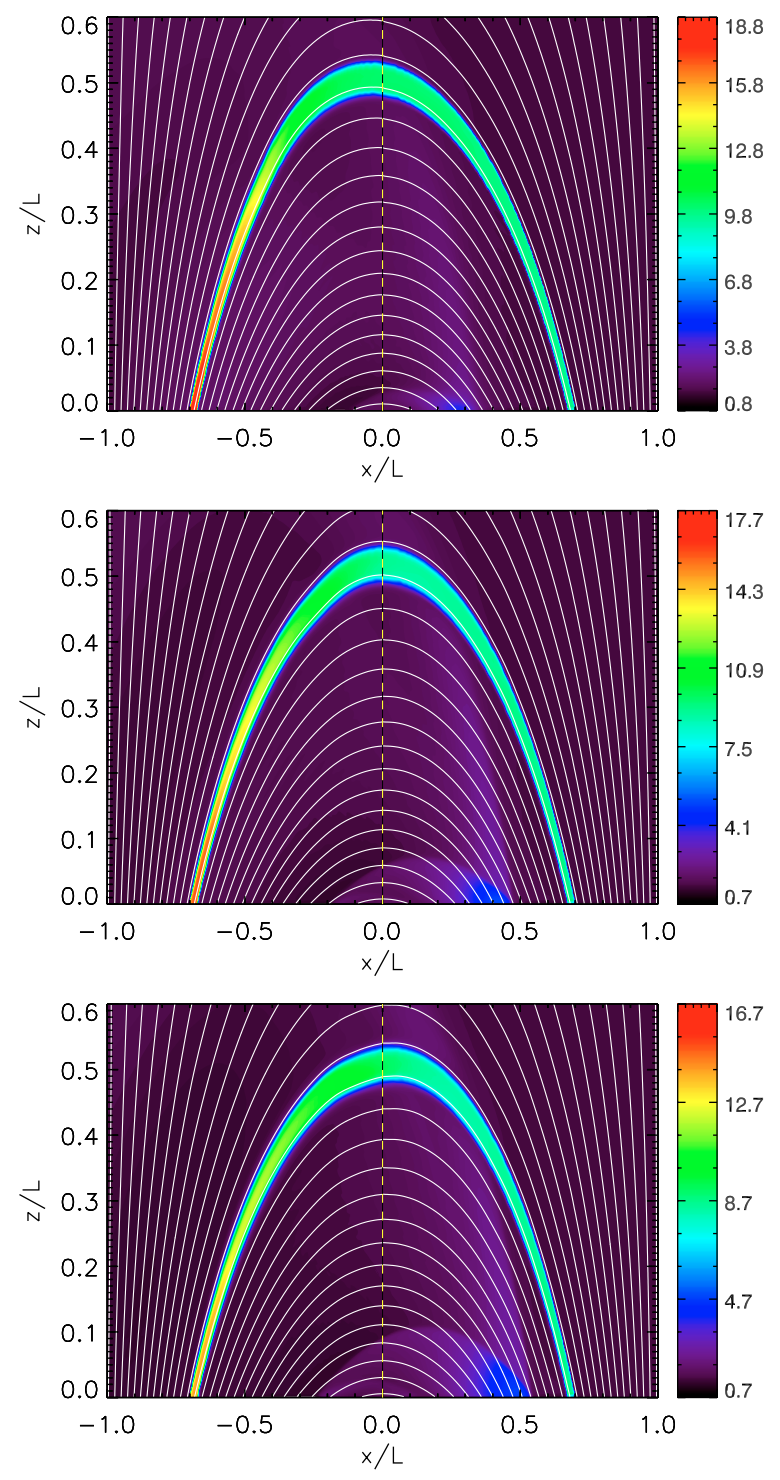

Fig. 6. Distribution of the mass density (colour scale in units of $\varrho_{\mathrm{e}}$ ) and magnetic field lines (white solid lines) for a pulse launched at $x_{0}=$ $-0.42 L, z_{0}=0$ for $t=200 \mathrm{~s} \mathrm{(top} \mathrm{panel),} t=300 \mathrm{~s}$ (middle panel) and $t=375 \mathrm{~s}$ (bottom panel). Vertical dashed yellow line corresponds to $x_{0}=0$.

somewhat above the foot-point, leading to roughly a $15 \%$ reduction in propagation time for the slow mode. Due to the finite width of the pulse the first disturbance at the loop's apex is seen at around $\left(0.9 l-w / 2 c_{\mathrm{s}}\right) \approx 700 \mathrm{~s}$. Comparing with Fig. 5 we find these estimates to be in good agreement with the computations, confirming the identification of this density pulse in the loop with the slow mode.

Whereas for a pulse launched at $x_{0}=0, z_{0}=0$ the main force exerted on the loop is perpendicular to the field lines (so that predominantly transverse waves are excited, see below), a pulse launched close to one foot-point also accelerates gas along the field lines, giving rise to a slow-mode pulse traveling along the loop from the foot-point nearest to the initial location of the pulse. As demonstrated by Selwa et al. (2005a) an initial pulse launched asymmetrically inside a 1-D loop and closer to one foot-point efficiently generates the fundamental mode of slow standing waves, while a pulse launched symmetrically or almost symmetrically within a loop generates mainly the first harmonic of slow standing waves. 


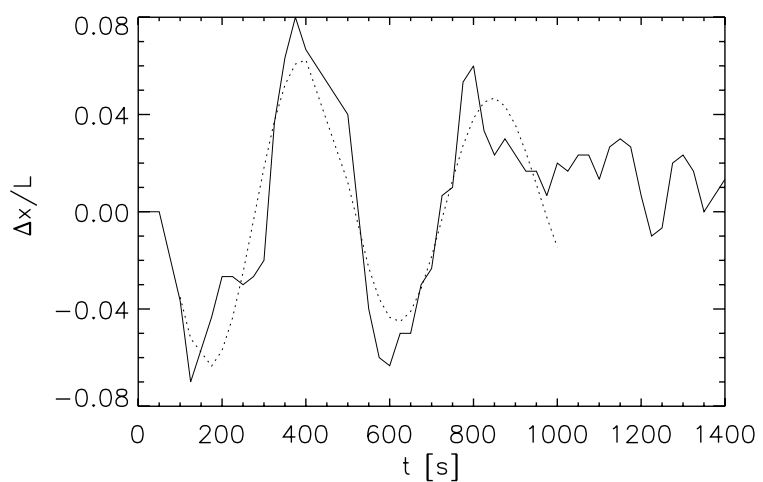

Fig. 7. The displacement of the apex along the $x$-axis for a pulse launched at $x_{0}=-0.42 L, z_{0}=0$ (solid line) together with a best-fit attenuated sine function (dotted line).
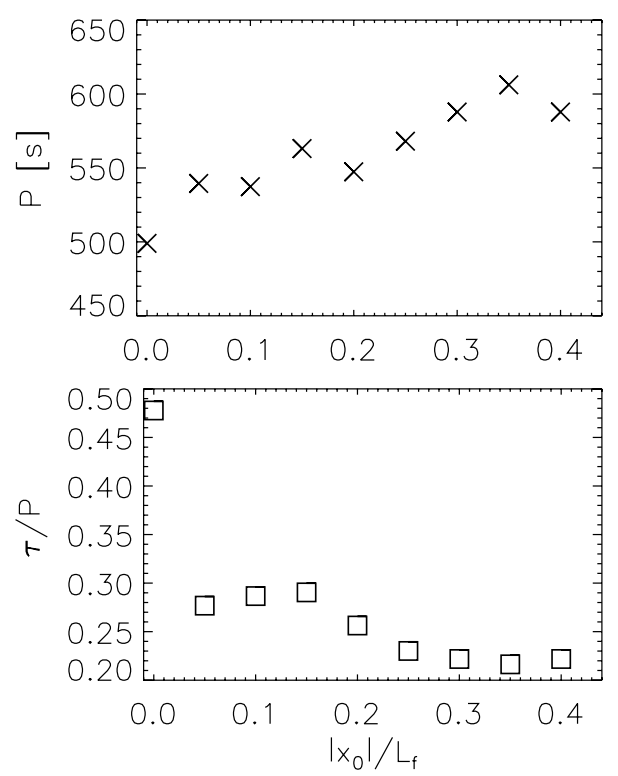

Fig. 8. Period $P$ (top panel) and the ratio of attenuation time $\tau$ to period, $\tau / P$ (bottom panel) of vertical oscillations vs. normalized pulse position $\left|x_{0}\right| / L_{\mathrm{f}}$.

It is noteworthy that mass is redistributed due to the slow wave from the location where the pulse hits the loop to the other end of the loop, i.e. from one foot-point to the other for asymmetric excitations, and from the apex to the foot-points for symmetric excitations. Such redistributions for symmetric and asymmetric pulses are confirmed by our 2-D simulations, as can be seen from Fig. 9. The material flows to the foot-points, also in the symmetric case (top panel of Fig. 9), is the slow mode speed.

A slow mode-like pulse is more clearly identified for larger values of $\left|x_{0}\right|$, for which the pulse propagates along the loop from one foot-point to another, rarefying one part of the loop and compressing another. For symmetric excitations $\left(x_{0}=0\right)$ basically mass is seen sliding from the apex to the foot-points. Another interesting feature is a density antinode (depression) located along $x=0$ only for a symmetric excitation (which is clearly visible as a lower density region in the top panel of Fig. 9 and is the cause of the density reduction with time in Fig. 2). In the case of asymmetric excitation such an antinode is observed as a rarefied region at one foot-point. As a consequence of the low value of the slow wave speed long numerical runs are required to set up a standing slow wave. Due to numerical instabilities which set in after some time the code crashed and we were not able to run
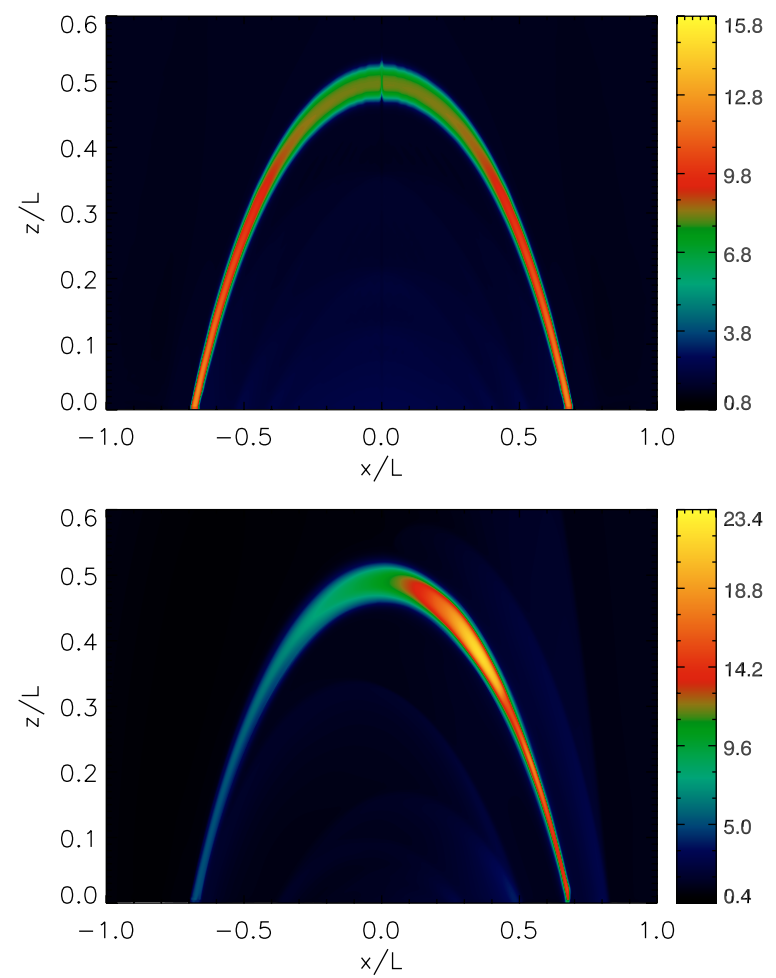

Fig. 9. Distribution of the mass density (colour scale in units of $\varrho_{\mathrm{e}}$ ) for a pulse launched at $x_{0}=z_{0}=0$ (top panel) and $x_{0}=-0.42 L, z_{0}=0$ (bottom panel) for $t=1400 \mathrm{~s}$.

the simulation for a sufficient time to set up standing slow mode oscillations. However, we do see a reflection of the pulse at a foot-point in some simulations. This, together with the fact that the pulse propagates at the slow-mode speed, supports the conclusion that a slow mode may be excited by the external pulse. Note the increase of density of such a mode comparing to initial configuration (Fig. 1).

\subsection{Dependence on pulse position: pulses along the vertical line $x_{0}=0$}

Now we fix the horizontal position of the initial pulse at $x_{0}=0$ and change its vertical position, i.e. we vary $z_{0}$. The top panel of Fig. 10 shows time-signatures of the loop's apex produced by an initial pulse launched at $x_{0}=0$ and $z_{0}=0.25 \mathrm{~L}$. By comparing this figure with the top panel of Fig. 2, which shows oscillations excited by a pulse that is identical except for its location, we infer that more oscillation periods are visible in the case of a pulse launched above the line $z_{0}=0$. This does not a priori mean that the wave is less strongly attenuated. As we shall see later in this section the opposite is the case. More peaks are seen because the amplitude of the excited oscillation is larger compared with the thickness of the loop and the amplitude of the fast mode (see Sect. 3.5). This is caused by wave reflection from the bottom boundary, which is line-tied, so that a larger amount of energy is deposited in the loop. In fact, the oscillation shown in the top panel of Fig. 10 looks rather similar to that in the lower panel of Fig. 2 (and both turn out to have a very similar $\tau / P$ ratio). In general, a pulse launched closer to the loop causes a larger initial shift of the loop (top panel of Fig. 11), although at large $z_{0} / L$ the excited oscillation amplitude saturates.

Middle and bottom panels of Fig. 11 display wave pe$\operatorname{riod} P$ and ratio $P / \tau$ vs. normalized pulse position $z_{0} / L_{\mathrm{f}}$. The 

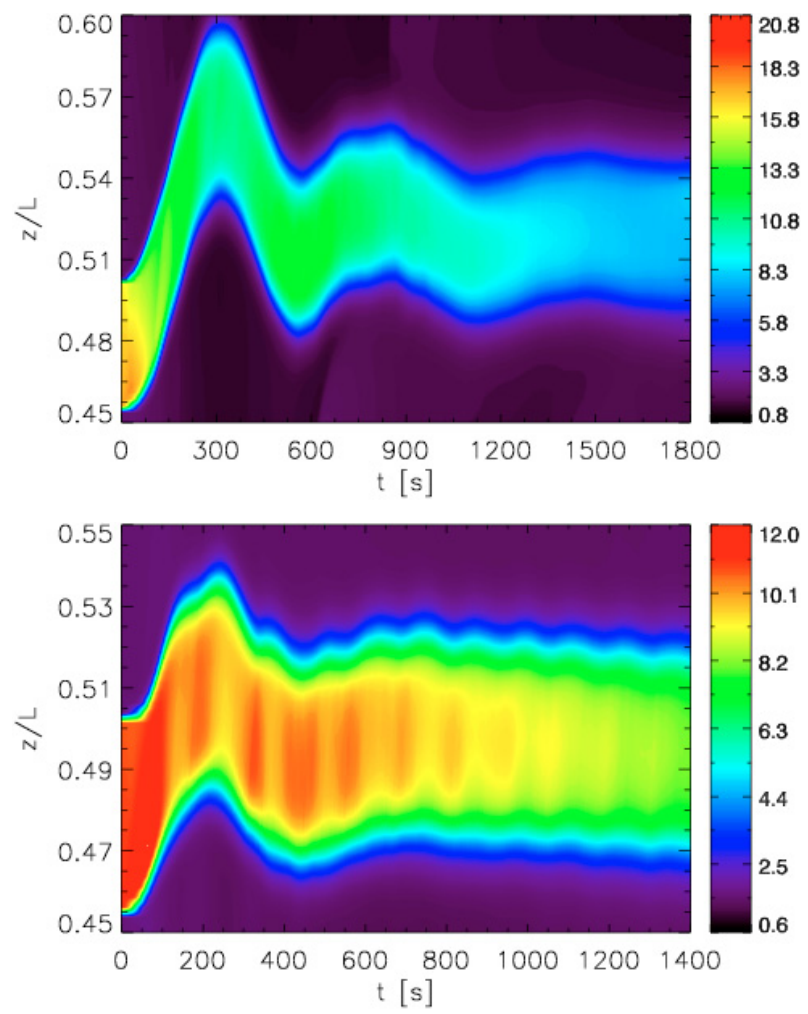

Fig. 10. Time-signature of the mass density (colour scale; arbitrary units: $10^{-12} \mathrm{~kg} \mathrm{~m}^{-3}$ ) for an initial pulse launched on the line $x_{0}=0$ at $z_{0}=0.25 \mathrm{~L}$ with a width of $w=0.35 \mathrm{~L}$ (top panel) and $x_{0}=z_{0}=0$ with $w=0.28 L$ (bottom panel). Compare with the top panel of Fig. 2 for which $z_{0}=0$ and $w=0.35 \mathrm{~L}$.

position of the loop's apex corresponds to $0.343 L_{\mathrm{f}}$. The general trends in $P$ and $P / \tau$ are similar to those seen in Fig. 8: $P$ grows while $\tau / P$ essentially declines with $z_{0}$. The growth of $P$ with $z_{0}$ follows from the fact that a pulse launched closer to the loop's apex transmits more energy into the loop, stretching it more and shifting its summit to higher levels. As a result the loop does not return to its initial position with an offset higher for pulses launched closer to the apex. Such stretched loops oscillate with longer waveperiods. The decrease of $\tau / P$ with increasing $P$ is in qualitative agreement with the behaviour seen in bottom panels of Figs. 3 and 8.

\subsection{Pulses of various widths}

We consider now pulses launched at $x_{0}=z_{0}=0$, but allow their width $w$ (and strength since the amplitude is constant) to vary. Bottom panel of Fig. 10 illustrates the corresponding timesignatures of the mass density for a pulse width of $w=28 \mathrm{Mm}$. Comparing this figure with the top panel of Fig. 2 which corresponds to $w=35 \mathrm{Mm}$ we conclude that the apex is shifted to higher levels for wider pulses due basically to the larger pulse energy. The shift increases with the amount of energy deposited in the loop (top panel of Fig. 12).

Middle and bottom panels of Fig. 12 show wave period $P$ and $P / \tau$ of the vertical kink mode vs. normalized pulse width $w / L_{\mathrm{f}}$. Again, this may serve as evidence that the attenuation mechanism is due to energy leakage: wider (and therefore stronger) pulses stretch the loop more and increase its curvature. The fundamental mode vertical oscillations are then less efficiently trapped by the loop structure, resulting in energy leakage into the ambient medium.
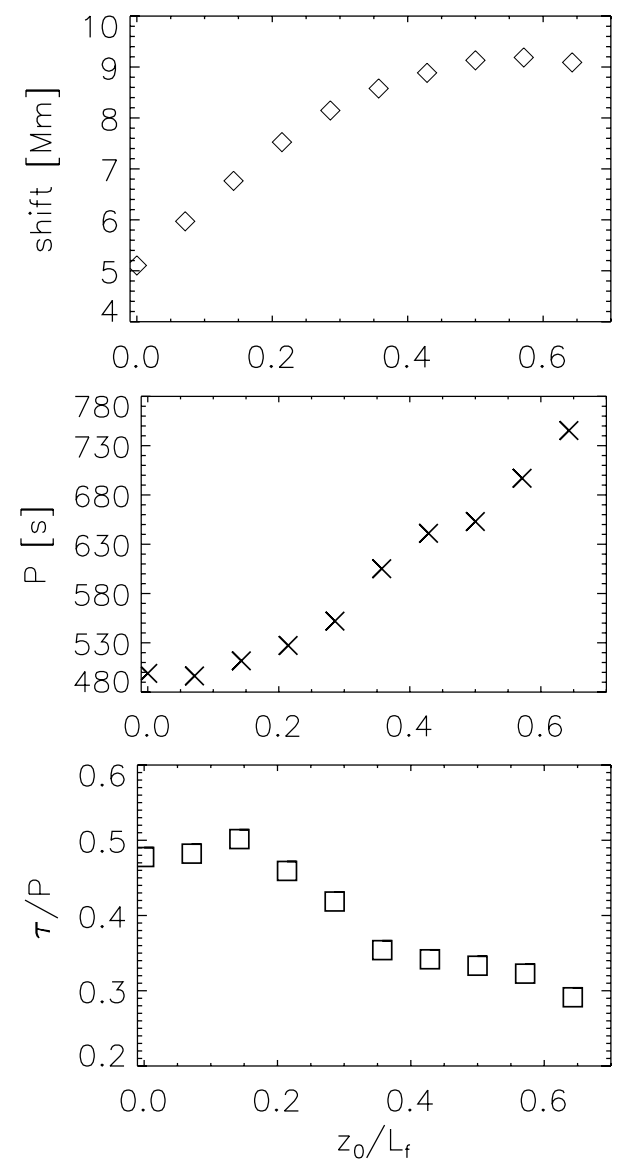

Fig. 11. Initial vertical shift of the loop's apex (top panel), period $P$ (middle panel) and the ratio of attenuation time $\tau$ to period, $\tau / P$ (bottom panel) of vertical oscillations vs. normalized pulse position $z_{0} / L_{\mathrm{f}}$.

\subsection{Fast propagating modes}

In all the studied cases a fast propagating mode is set up. The initial amplitude of fast propagating waves is about $0.0022 \mathrm{~L}$ for all tested pulse amplitudes and widths. Thus the fast mode amplitude appears to be relatively independent of the energy of the external pulse, whereas the standing kink mode amplitude depends strongly on these parameters. Consequently, the ratio of fast mode amplitude to the vertical kink mode amplitude, which is $A_{\text {fast }} / A_{\text {kink }}=0.043$ for a pulse initially located at $x_{0}=z_{0}=0$, with $w=0.35 L, A_{\mathrm{p}}=15 p_{\mathrm{e}}$, also depends on the energy of the external pulse. This explains why the fast mode is better seen in figures with lower kink amplitudes (bottom panel of Fig. 10 and top panel of Fig. 2) than in those with large amplitude vertical kink modes (top panel of Fig. 10 and bottom panel of Fig. 2). As the location of the initial pulse is moved away from $x_{0}=z_{0}=0$ towards one of the foot-points of the loop, the amplitude of the excited fast mode decreases and becomes increasingly difficult to separate from the signals due to the other modes. Fast mode oscillations can be more clearly seen in mass density (Fig. 13) than in displacement. Note that density changes due to the standing kink mode and the slow mode/longterm trend were removed prior to plotting Fig. 13.

We can determine the period (plotted in the top panel of Fig. 14 vs. pulse width) and the attenuation time of the fast oscillations (bottom panel of Fig. 14) by fitting attenuated sine functions to the fast oscillations time signatures (e.g. shown in Fig. 13). Like the period of the vertical kink oscillations, the period of the fast oscillations grows with pulse width, i.e. pulse 

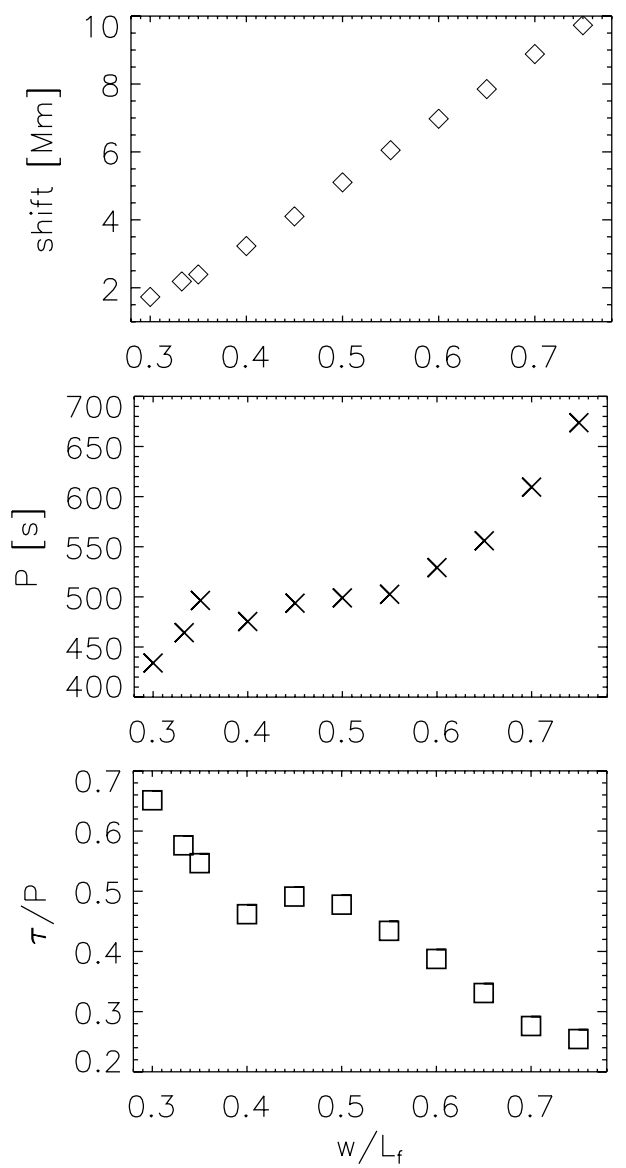

Fig. 12. Initial shift of the loop's apex (top panel) period $P$ (middle panel) and the ratio of attenuation time $\tau$ to the period, $\tau / P$, (bottom panel) vs. normalized pulse width $w / L_{\mathrm{f}}$.

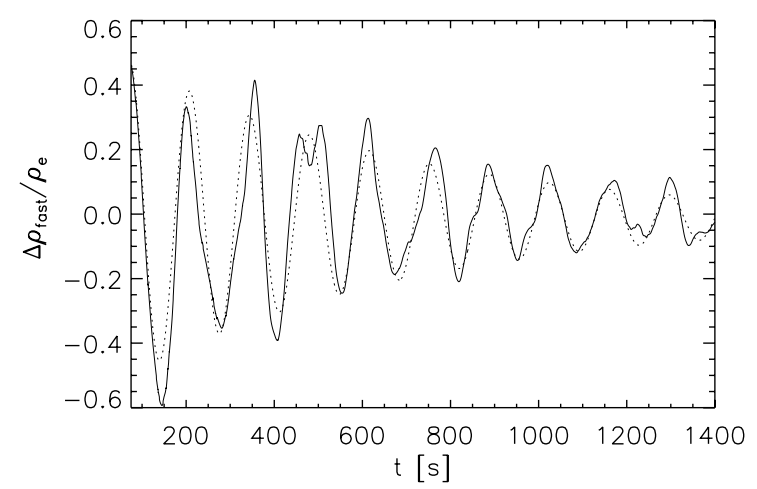

Fig. 13. Fast oscillations seen in mass density at the loop's apex (solid line) together with the best fit attenuated sine function (dotted line) for the pulse launched at $x_{0}=z_{0}=0$ with $w=0.35 \mathrm{~L}$.

energy (top panel of Fig. 14), while the attenuation time of fast oscillations generally decreases with pulse width (bottom panel of Fig. 14), although this dependence is less clear.

\subsection{Attenuation of vertical oscillations}

We expect that larger period waves are more weakly attenuated by a classical attenuation phenomenon like viscosity. As the applied MHD equations are ideal and numerical diffusion is small, viscosity is practically not present here and the mechanism of wave attenuation acting in these simulations differs from viscous
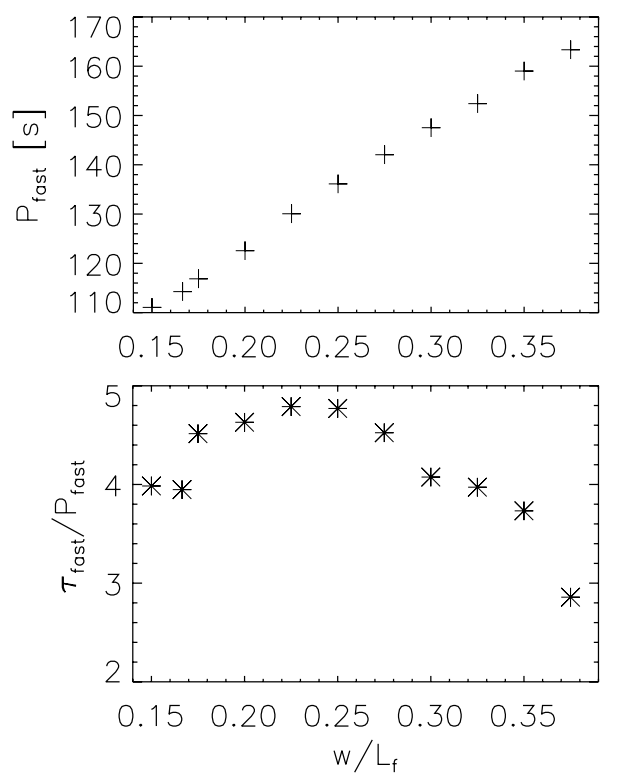

Fig. 14. Period of the fast oscillations $P_{\text {fast }}$ (top panel) and the ratio of attenuation time of the fast oscillations $\tau_{\text {fast }}$ to the period of the fast oscillations, $\tau_{\text {fast }} / P$, (bottom panel) vs. normalized pulse width, $w / L_{\mathrm{f}}$.

attenuation. Here we briefly explore the conjecture that attenuation is due to energy leakage. Longer wavelength waves experience more difficulties in fitting into a curved loop structure and as a result they leak energy into the ambient medium (Wentzel 1974). This process leads to a decrease of the wave amplitude - a process which is characteristic of wave attenuation. These findings are in general agreement with the results of Murawski \& Roberts (1993), who studied energy leakage of normal modes in coronal structures.

Evidence of energy leakage from the loop is provided by perturbed energy density profiles (Fig. 15). The position of the loop corresponds to the white contour at the bottom of each frame in this figure. The first maximum from the top, represented by a pink-white patch, results from the initial pulse, but other maxima are a consequence of the energy leakage (red areas outside the loop). Additional evidence that energy leaks from the loop via fast magnetosonic waves is provided by the fact that the perturbations in the thermal and magnetic pressures are in-phase outside the loop. A more detailed analysis and discussion will be given in a separate paper (Selwa et al. 2006).

\section{Summary and discussion}

The results we obtained in this paper can be summarized as follows. Firstly, an external pulse emitted below a loop embedded in an arcade excites different wave modes, with the properties of these waves depending on the location of the pulse and on its strength.

The most prominently visible mode is a vertical kink-like standing mode, which bears many of the properties of the vertical oscillation observed by Wang \& Solanki (2004). In particular, the simulation reproduces both the observed period and amplitude (thus improving on the work of Murawski et al. 2005a,b; and Selwa et al. 2005b). In addition, higher frequency fast magnetosonic waves are excited in most cases, although their signatures are less clear when the pulse is located close to a foot-point of the loop. Finally, a slow mode pulse is excited in the loop when the external pulse is located close to a loop foot-point. The slow-mode pulse propagates towards the other foot-point, 

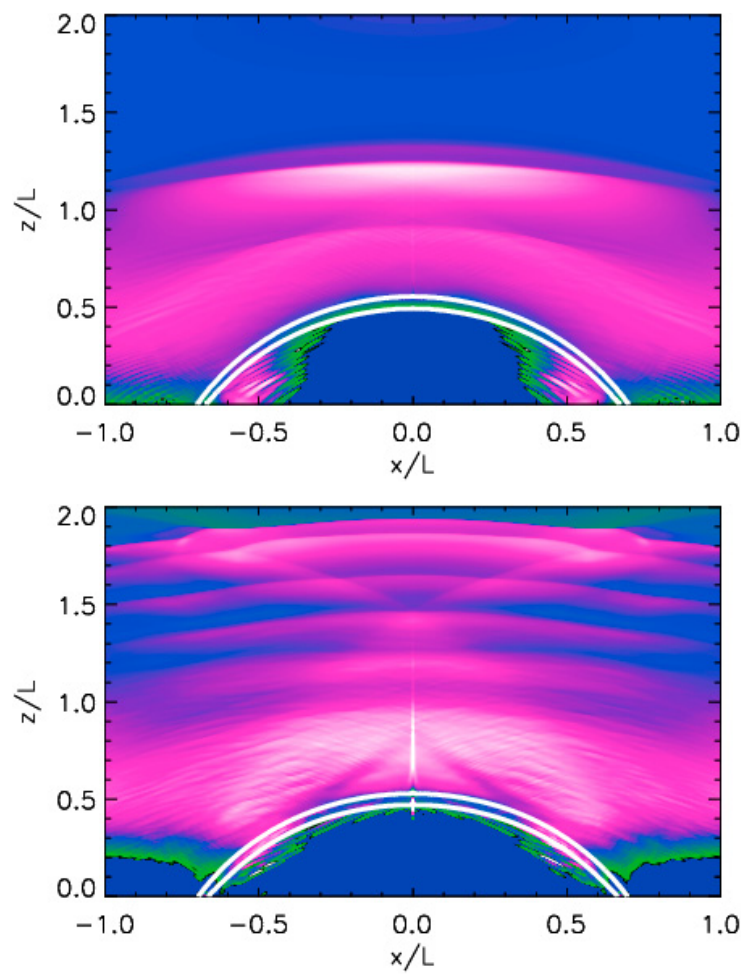

Fig. 15. Evolution of perturbed energy beyond the loop showing energy leakage ( $t=300 \mathrm{~s}-$ top panel, $t=1300 \mathrm{~s}-$ bottom panel $)$ in the case of $A_{\mathrm{p}}=15 p_{\mathrm{e}}$ and $x_{0}=z_{0}=0$.

similar to the pulses seen by Wang et al. (2005) in SUMER observations and by Selwa et al. (2005a) in 1-D simulations. Interestingly, when the exciting pulse is located directly below the loop's apex, matter flows down both legs of the loop (until being reflected), consistent with the setting up of the first harmonic slow mode in the loop. This agrees well with the findings of Selwa et al. (2005a), that the first harmonic is excited when the excitation occurs close to the loop's apex.

The vertical kink mode is associated with an asymptotic net displacement of the plasma column, which persists until the end of the simulations, well after the vertical kink oscillations have died away. The column is displaced as the whole loop structure attains a different equilibrium.

It is noteworthy that irrespective of the pulse position only the fundamental mode of vertical kink oscillations is excited. According to Roberts et al. (1984) the period of the kink mode, $P$, depends of the Alfvén speed and the length of the loop, $l$ :

$P=\frac{2 l}{n V_{\mathrm{A}}}$,

where $V_{\mathrm{A}}$ denotes the average Alfvén speed such that $V_{\mathrm{Ai}} \leq$ $V_{\mathrm{A}} \leq V_{\mathrm{Ae}}$ and $n$ is the number of the mode. As the reference value we use the fundamental mode with centrally located pulse. As the Alfvén speed varies with height, we can estimate average $V_{\mathrm{A}}$ along the loop from Eq. (15). Note, that for the centrally located pulse $P \sim 500 \mathrm{~s}$, so if we suppose that the length of the loop remains unchanged, the period of the first harmonic should be $P / 2 \sim 250 \mathrm{~s}$ and of the second harmonic $P / 3 \sim 167 \mathrm{~s}$, respectively. As time-signatures we studied are rather smooth (there are no signs of $\sim 250 \mathrm{~s}$ oscillations) and damped sine function can be easily fit, we conclude that no first harmonic is observed, even in the case of asymetrically located initial pulse.
There are also further conclusions: $\tau / P$ remains smaller than observed, irrespective of the chosen parameters. This suggests that there are still some significant differences between observed and modeled loops. We find that a pulse near $x_{0}=0$ is needed to produce vertical loop oscillations that can be clearly observed. Since the pulse must be launched below the loop itself (in 3-D geometry) for a vertical oscillation, the choice of location of the pulse is quite limited. This may well be one reason why vertical oscillations of loops were not discovered earlier: they may be rare. We find, however, that for large $\left|x_{0}\right|$ the loop oscillates with a distortion mode, which to our knowledge has not been seen before in numerical simulations of loop oscillations.

A number of improvements to this study are possible. The most obvious is the inclusion of gravity (as studied for slow modes by Mendoza-Briceño et al. 2004). This will remove the artificial decrease of the Alfvén speed with height, which plagues the current model. The detailed study of different attenuation mechanisms will be given in a separate paper.

Acknowledgements. MS thanks Dr. Robert Cameron for his comments and helpful discussions. KM expresses his sincere thanks to Dr. Luigi Nocera for stimulating discussions. The authors express their thanks to the unknown referee for his/her stimulating comment. The magnetohydrodynamics code used in this study was developed at the University of Washington by Ogden S. Jones, Uri Shumlak, Scott Eberhardt, Bogdan Udrea, and provided through the sponsorship of AFOSR program. MS's \& KM's work was financially supported by a grant from the State Committee for Scientific Research Republic of Poland, with KBN grant No. 2 PO3D 01625.

\section{References}

Andries, J., Goossens, M., Hollweg, J. V., Arregui, I., \& Van Doorsselaere, T. 2005, A\&A, 430, 1109

Aschwanden, M., Fletcher, L., Schrijver, C., \& Alexander, D. 1999, ApJ, 520, 880

Brady, C. S., \& Arber, T. D. 2005, A\&A, 438, 733

Cadez, V. M., Oliver, R., \& Ballester, J. L. 1996, A\&A, 314, 636

del Zanna, L., Schaekens, E., \& Velli, M. 2005, A\&A, 431, 1095

de Moortel, I., Ireland, J., Walsh, R. W., \& Hood, A. W. 2002, Sol. Phys., 209, 61

Edwin, P. M., \& Roberts, B. 1983, Sol. Phys., 88, 179

Jones, O. S., Shumlak, U., \& Eberhardt, D. S. 1997, J. Comput. Phys., 130, 231

Keppens, R., \& Tóth, G. 1999, Phys. Plasmas, 6(5), 1461

Mendoza-Briceño, C. A., Erdélyi, R., Sigalotti, L., \& Di, G. 2004, ApJ, 605, 493

Murawski, K., \& Roberts, B. 1993, Sol. Phys., 143, 89

Murawski, K., Selwa, M., \& Rossmanith, J. A. 2005a, Sol. Phys., 231, 87

Murawski, K., Selwa, M., \& Nocera, L. 2005b, A\&A, 437, 687

Nakariakov, V. M. 2003, in The Dynamic Sun, ed. B. Dwivedi (CUP)

Nakariakov, V. M., Ofman, L., Deluca, E. E., Roberts, B., \& Davila, J. M. 1999, Science, 285,862

Oliver, R., Hood, A. W., \& Priest, E. R. 1996, ApJ, 461, 424

Oliver, R., Murawski, K., \& Ballester, J. L. 1998, A\&A, 330, 726

Roberts, B., Edwin, P. M., \& Benz, A. O. 1984, ApJ, 279, 857

Ruderman, M. S., \& Roberts, B. 2002, ApJ, 577, 475

Sedlacek, Z. 1971, J. Plasma Phys., 5, 239

Selwa, M., Murawski, K., \& Solanki, S. K. 2005a, A\&A, 436, 701

Selwa, M., Murawski, K., Solanki, S. K., Wang, T. J., \& Tóth, G. 2005b, A\&A, 440,385

Selwa, M., Murawski, K., Solanki, S. K., \& Wang, T. J. 2006, A\&A, submitted

Smith, J. M., Roberts, B., \& Oliver, R. 1997, A\&A, 317, 752

Terradas, J., Oliver, R., \& Ballester, J. L. 1999, ApJ, 517, 488

Van Doorsselaere, T., Andries, J., Poedts, S., \& Goossens, M. 2004b, ApJ, 606, 1223

Van Doorsselaere, T., Debosscher, A., Andries, J., \& Poedts, S. 2004b, A\&A, 424,1065

Ye, H., Sedlacek, Z., \& Mahajan, S. M. 1993, Phys. Fluids, B5, 2999

Wang, T. J., Solanki, S. K., Innes, D. E., \& Curdt, W. 2005, A\&A, 435, 753

Wang, T. J., \& Solanki, S. K. 2004, A\&A, 421, L33

Wang, T. J., Solanki, S. K., Curdt, W., Innes, D. E., \& Dammash, I. E. 2002, ApJ, 574, L101

Wentzel, D. G. 1974, Sol. Phys., 39, 129 